\title{
Long-Term Self-Reported Cognitive Problems After Delirium in the Intensive Care Unit and the Effect of Systemic Inflammation
}

\author{
Annemiek E. Wolters, MD, MSc, * Linda M. Peelen, PhD, *广 Dieuwke S. Veldhuijzen, PhD, *t \\ Irene J. Zaal, MD, PhD, *Dylan W. de Lange, MD, PhD, * Wietze Pasma, DVM, * \\ Diederik van Dijk, MD, PhD, * Olaf L. Cremer, MD, PhD, * and Arjen J. C. Slooter, MD, PhD*
}

OBJECTIVES: To describe the association between intensive care unit (ICU) delirium and self-reported cognitive problems in 1-year ICU survivors, and investigate whether this association was altered by exposure to systemic inflammation during ICU stay.

DESIGN: Prospective cohort study.

SETTING: Dutch medical-surgical ICU.

PARTICIPANTS: One-year ICU survivors, admitted to the ICU $\geq 48$ hours.

MEASUREMENTS: Self-reported cognitive problems were measured with the Cognitive Failures Questionnaire (CFQ). Cumulative exposure to systemic inflammation was based on all daily C-reactive protein (CRP) measurements during ICU stay, expressed as the area under the curve (AUC). Multivariable linear regression was conducted to evaluate the association between delirium and the CFQ. The effect of inflammation on the association between delirium and CFQ was assessed, comparing the effect estimate (B) of delirium and CFQ between models with and without inclusion of the AUC of CRP.

RESULTS: Among 567 1-year ICU survivors, the CFQ was completed by 363 subjects. Subjects with multiple days of delirium during ICU stay reported more selfreported cognitive problems $(\mathrm{Badj}=5.10,95 \%$ CI 1.01 9.20), whereas a single day delirium was not associated with higher CFQ scores $(\mathrm{Badj}=-0.72,95 \% \mathrm{CI}-5.75$ to 4.31). Including the AUC of CRP did not change the association between delirium and the CFQ (ratio for a single

From the *Department of Intensive Care Medicine, University Medical Center Utrecht, Heidelberglaan 100, 3508 GA, Utrecht, The

Netherlands; ‘Department of Epidemiology, Julius Center for Health Sciences and Primary Care, University Medical Center Utrecht, Utrecht, The Netherlands; and Institute of Psychology, Health, Medical, and Neuropsychology Unit, Leiden University, Leiden, The Netherlands.

Related paper presentations: Results of this study have been presented at the 10th EDA/BGS Meeting, September 2015.

Address correspondence to Annemiek E. Wolters, Department of Intensive Care Medicine, Room F.06.149, 3508 GA Utrecht, the Netherlands.

E-mail: a.e.wolters@umcutrecht.nl

DOI: $10.1111 /$ jgs. 14660

and multiple days were respectively: $1.00,95 \%$ CI 0.59 1.44 and $0.86,95 \%$ CI $0.47-1.16$ ).

CONCLUSION: Multiple days of delirium was associated with long-term self-reported cognitive problems. The cumulative exposure to systemic inflammation did not alter this association, suggesting that delirium in the context of little inflammation is also detrimental. J Am Geriatr Soc 65:786-791, 2017.

Key words: delirium; long-term self-reported cognitive problems; systemic inflammation; C-reactive protein; intensive care unit

$\mathbf{I}_{\mathrm{t} i \mathrm{i}}^{\mathrm{n}}$ ntensive care unit (ICU) survivors experience a substantial amount of long-term morbidity, including problems in cognitive functioning. ${ }^{1,2}$ A pivotal risk factor for longterm cognitive problems after ICU stay seems to be the occurrence of delirium during critical illness. ${ }^{3-6}$ Delirium has a multifactorial pathophysiology, and it is usually not possible to ascertain one specific cause of delirium in ICU patients. Previous studies found acute systemic inflammation to be a risk factor for delirium ${ }^{7-9}$ and also for long-term cognitive impairment. ${ }^{2,10}$ In rodent studies, neuroinflammation and neuronal loss was seen up to 10 months after systemic inflammation, induced by one peripheral injection of lipopolysaccharide. ${ }^{11}$ Persistent neuroinflammation may thus explain why patients with exposure to acute systemic inflammation and resulting delirium develop long-term cognitive impairment. ${ }^{8}$ Delirium is, however, not exclusively associated with exposure to acute systemic inflammation and can also result from other causes, such as metabolic disturbances. Up until now, it was unclear whether the association between delirium and long-term self-reported cognitive problems was mediated by the degree of exposure to acute systemic inflammation. ${ }^{8,9}$

Recent literature shows that rapidly-reversible sedation-related delirium has a better outcome than persistent 
delirium. ${ }^{12}$ Although unstudied, it is plausible, that the outcome of a very brief episode of delirium is also favorable, as compared to more delirium days in terms of cognition. The aim of this study was to investigate the association of a single day or multiple days of ICU delirium with long-term self-reported cognitive problems in 1-year ICU survivors, and to assess whether this association was mediated by exposure to systemic inflammation during ICU stay.

\section{METHODS}

\section{Setting and Study Population}

This prospective, observational study was conducted in consecutively admitted adults, who stayed for at least 48 hours in the 32-bed mixed medical-surgical ICU of the University Medical Center Utrecht (UMCU) between January 2011 and June 2013, and who were alive 1 year after discharge. Survival status was determined by a query of the Dutch municipal register. Excluded were subjects who were transferred from an ICU of another hospital, as well as subjects with an acute neurological illness or another condition that could hamper delirium assessment. The Medical Research Ethics Committee of the UMCU waived the need for informed consent (protocols 12/421, 10/056 and 10/006).

\section{Data Collection}

A detailed description of the daily assessment of mental status was recently published..$^{13}$ In brief, an algorithm was used to evaluate daily mental status of each patient during the preceding 24 hours (awake without delirium, delirium, or coma). For this assessment, the Richmond Agitation Sedation Scale (RASS) $^{14}$ and Confusion Assessment Method for the ICU (CAM-ICU) ${ }^{15}$ scores from the bedside nurses were used, next to initiation of treatment with neuroleptic drugs in the previous 24 hours, chart review, and an additional CAM-ICU by trained research nurses. ${ }^{13}$

All subjects who were alive 1 year after ICU discharge received a survey by mail containing multiple questionnaires, including the validated Dutch translation of the Cognitive Failures Questionnaire (CFQ), ${ }^{16,17}$ which has been used as a long term outcome in postoperative care and critical illness populations before. ${ }^{18,19}$ The CFQ consists of 25 questions, scored on a 5-point Likert scale, and measures self-reported frequencies of failures in attention, memory, and action. The total score on the CFQ ranges from 0 to 100 , with a higher score indicating more selfreported cognitive problems. ${ }^{17} \mathrm{~A}$ reminder was sent and follow-up phone calls were made when subjects did not respond.

Acute systemic inflammation during ICU stay was quantified by daily C-reactive protein (CRP) levels (in milligrams per liter $[\mathrm{mg} / \mathrm{L}])$. CRP is an acute-phase reactant found to be elevated in blood in inflammatory and infectious conditions. ${ }^{20}$ CRP levels in the ICU were measured at admission and daily thereafter. Daily CRP levels were used to calculate the area under the curve (AUC in mg*days $(\mathrm{L})$, as a measure of total exposure to acute systemic inflammation during ICU stay. ${ }^{21}$
At ICU admission, we recorded data on age, gender, chronic disease burden according to the Charlson Comorbidity Index (CCI), ${ }^{22}$ and the Acute Physiology And Chronic Health Evaluation IV (APACHE IV) as a measure for severity of illness in the first 24 hours of ICU admission. ${ }^{23}$ Severity of illness on subsequent days was assessed by the Sequential Organ Failure Assessment (SOFA) ${ }^{24}$ score.

\section{Statistical Methodology}

To assess whether delirium during ICU stay was an independent risk factor for long-term self-reported cognitive problems, multivariable linear regression models were used. Results of these analyses were presented as unstandardized regression coefficients (B), with accompanying $95 \%$ confidence intervals $(95 \% \mathrm{CI})$. The presence of delirium was classified into three groups: (1) subjects with no delirium during ICU admission (used as the reference group), (2) subjects with a single day of ICU delirium, and (3) subjects with multiple days of delirium during ICU stay. In multivariable linear regression models, adjustments were made for covariables with the potency to be a confounder in the association between delirium and long-term self-reported cognitive problems. These covariables were chosen a priori based on clinical judgment and a recent systematic review, ${ }^{25}$ and were age, gender, CCI and the APACHE IV. Further, the maximal total SOFA score (leaving out the central nervous system component to prevent indirect adjustment for the presence of delirium) was entered in the models as a measure for severity of illness during ICU stay. Trend imputation was used for missing values of daily measured variables, including the missing levels of CRP, given the availability of longitudinal data both preceding and following each measurement. ${ }^{26}$

Additional analyses were conducted to assess to which extent the degree of acute systemic inflammation contributed to the association between delirium and long-term self-reported cognitive problems. To this end, we used the following approach. From our study population 1,000 bootstrap samples were drawn with replacement. Within each bootstrap sample two models were developed: a model as described in the previous paragraph, including all potential confounders (Model 1) and a model containing the same variables plus the amount of inflammation during ICU stay (expressed as the AUC of the daily CRP levels) (Model 2). The effect estimates indicating the association between delirium (single and multiple days) and CFQ resulting from both models were compared by calculating their ratio and their absolute difference. Subsequently, these ratios were averaged over the bootstrap samples to obtain an overall estimate; a 95\% CI around these estimates was obtained non-parametrically. The overall estimate for the absolute difference with accompanying 95\% CI was calculated in a similar fashion. If the ratios were statistically significant different from 1 or the absolute differences were statistically significant different from 0 , the amount of acute systemic inflammation could be considered to contribute to the association between delirium and CFQ. ${ }^{27}$

In our cohort, subjects with at least one missing value in the CFQ were different from those with complete CFQ 
data with regard to age, gender, CCI and maximal SOFA score (Supplementary Appendix S1). Missing data rarely occurs entirely at random, and excluding these subjects could potentially lead to biased results. ${ }^{26,28}$ Therefore, next to analyzing data restricted to subjects who completed the full CFQ (i.e. complete case analysis) we also performed multiple imputation to assign values to missing answers to the questions of the CFQ in the 1-year ICU survivors, and analyzed the imputed dataset as a secondary analysis. ${ }^{28}$ For multiple imputation we conducted 10 iterations and used subjects and ICU-specific variables (e.g., gender, age, comorbidity score, severity of illness measures, and length of ICU stay), as well as answers to other questionnaires that were also included in the survey as predictors in the imputation model.

All data analyses were performed using IBM SPSS Statistics 21.0 for Windows and R version 3.1.1 for Windows ( $\mathrm{R}$ Foundation for Statistical Computing Vienna, Austria). A significance level of 0.05 was used for all statistical inferences and the null hypotheses were tested against two-sided alternatives.

\section{RESULTS}

Between January 2011 and June 2013, 2,669 subjects were screened for delirium of whom eventually 567 persons were eligible for this study (i.e., the 1-year ICU survivors). Application of the exclusion criteria is described in Figure 1 . In total, 546 subjects received a survey, of whom 402 individuals answered to the CFQ questionnaire (response rate $74 \%$ ), and 363 (90\% of the responders) completed the full CFQ (i.e. complete cases). These 363 subjects reported their CFQ scores after a median of 400 days (interquartile range [IQR] 380-415) following ICU discharge. Their characteristics are described in Table 1. Characteristics and differences between complete cases, subjects who completed the CFQ partially (i.e. partial responders), subjects who did not respond (i.e. nonresponders), and nonreceivers are outlined in Supplementary Appendix S1.

Intensive care unit delirium was present in $186(51 \%)$ of the 363 patients, and the median duration of delirium when delirium was present was 3 days (IQR $1-5$ ). The median CFQ scores for subjects who never had delirium, those who had a single day of delirium, and subjects with multiple days of delirium were 23 (IQR 12-34), 20 (IQR 10-31) and 25 (IQR 16-35), respectively. Multivariable linear regression with adjustment for potential confounders showed that a single day of ICU delirium was not associated with worse CFQ scores, as compared to subjects with no delirium (adjusted $\mathrm{B}=-0.72,95 \% \mathrm{CI}-5.75-4.31$, Table 2 ). In contrast, subjects with multiple ICU delirium days had more self-reported cognitive problems, than subjects with no delirium (adjusted $\mathrm{B}=5.10,95 \%$ CI 1.01-9.20, Table 2).

Of the daily measured CRP levels, 6\% was missing and was therefore imputed using longitudinal imputation. The median AUC of the CRP was 545 (IQR 259$1,029 \mathrm{mg} *$ days $/ \mathrm{L})$. The ratios of the effect estimates did not differ from 1 and the absolute differences were not different from 0 (Table 2), indicating that the AUC of the CRP levels were not contributing into the association between delirium and CFQ scores.

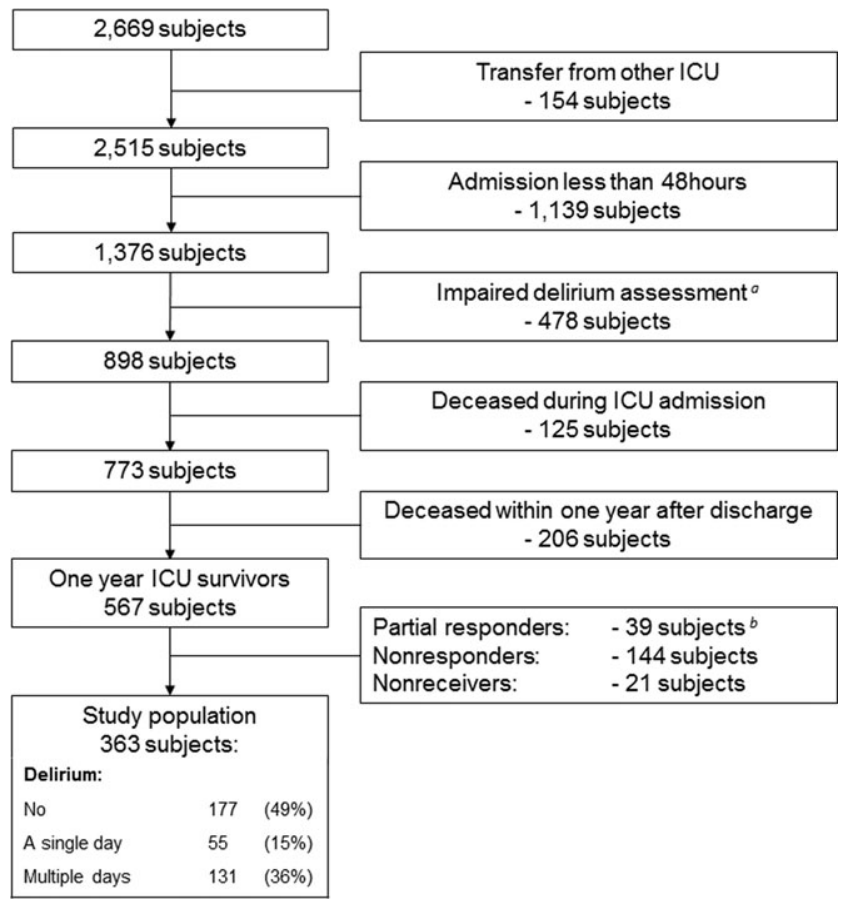

Figure 1. Study Flowchart. ${ }^{a}$ Neurological patients and/or other reasons for impaired delirium assessment e.g. mental retardation or language barrier. ${ }^{b}$ Partial responders sent the questionnaire back, with a partially completed CFQ. Nonresponders were subjects who did not sent the questionnaire back. To the nonreceivers we did not send a questionnaire because: they moved abroad $(\mathrm{n}=10)$, they died more than 1 year after discharge, but before the questionnaire was sent $(\mathrm{n}=6)$ or they declined any follow-up, and announced this in advance $(\mathrm{n}=5)$. ICU $=$ intensive care unit.

Although the distribution of the CFQ scores was altered after multiple imputation, as compared to the CFQ scores of the complete cases (higher versus lower scores and bimodal versus right skewed, respectively), multivariable linear regression analysis after multiple imputation showed similar results on both the association between delirium and CFQ, and the absence of a contributing effect of acute systemic inflammation (Supplementary Appendix S2).

\section{DISCUSSION}

In our study, we found that multiple days of ICU delirium was associated with long-term self-reported cognitive problems, in contrast to a single day of delirium. In addition, the association between delirium and long-term selfreported cognitive problems was not influenced by systemic inflammation during ICU stay. These findings suggest that not merely in the context of inflammation, but in the setting of other problems as well, delirium has worse long-term self-reported cognitive outcomes.

The association between delirium and long-term cognitive problems is consistent with previous research..$^{3-6}$ One earlier study investigated the association between inflammation during delirium onset and long-term selfreported cognitive problems measured with the CFQ. ${ }^{29}$ In this previous study, no association was found between 
Table 1. Characteristics of the Study Population

Delirium during ICU stay

Characteristic

$\begin{array}{cc}\text { No } & \text { Single day } \\ (n=177 ; 49 \%) & (n=55 ; 15 \%)\end{array}$

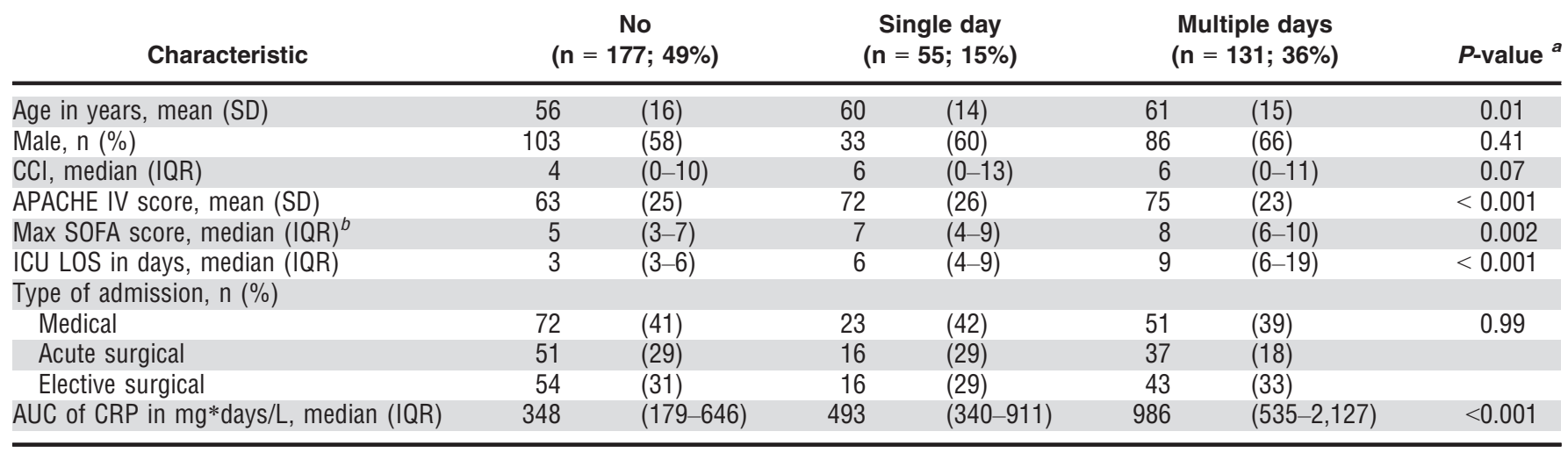

APACHE IV score $=$ Acute Physiology and Chronic Health Evaluation IV score; AUC = Area under the Curve; CCI = Charlson Comorbidity Index; Max SOFA = maximal total Sequential Organ Failure Assessment; ICU = Intensive Care Unit; IQR = Interquartile Range; LOS = Length of stay; SD = Standard Deviation; CRP = C-reactive Protein.

${ }^{a}$ Group comparisons were made using the Chi Square Test for categorical variables, one-way ANOVA for normally distributed continuous variables, or Kruskal-Wallis test for skewed distributed continuous variables.

${ }^{\mathrm{b}}$ Maximal total SOFA score without the neurological component.

Table 2. Association Between Delirium and Cognitive Failures Questionnaire (CFQ) and the Effect of Systemic Inflammation

Delirium during ICU stay

\begin{tabular}{|c|c|c|c|c|c|}
\hline Analysis & No & \multicolumn{2}{|c|}{ A single day } & \multicolumn{2}{|c|}{ Multiple days } \\
\hline \multicolumn{6}{|l|}{ Association between delirium and the $\mathrm{CFQ}^{a}$} \\
\hline Crude & Reference & -1.96 & $(-6.95$ to 3.03$)$ & 2.53 & $(-1.20$ to 6.26$)$ \\
\hline \multicolumn{6}{|c|}{ The effect of acute systemic inflammation on the association between delirium and $\mathrm{CFQ}^{\mathrm{C}}$} \\
\hline Ratio (95\% confidence interval) & - & 1.00 & $(0.59-1.44)$ & 0.86 & $(0.47-1.16)$ \\
\hline Difference (95\% confidence interval) & - & 0.01 & $(-0.20$ to 0.24$)$ & -0.87 & $(-2.26$ to 0.54$)$ \\
\hline
\end{tabular}

$\mathrm{CFQ}=$ Cognitive Failures Questionnaire.

$* P$-value $=0.02$.

${ }^{a}$ Unstandardized coefficient B with $95 \%$ confidence interval.

${ }^{\mathrm{b}}$ Adjusted for: age, gender, Charlson Comorbidity Index, Acute Physiology and Chronic Health Evaluation IV score, and maximal total Sequential Organ Failure Assessment without the neurological component.

${ }^{c}$ Ratio and absolute difference between the effect estimates indicating the association between delirium and CFQ adjusted for the aforementioned covariables (Model 1), and the effect estimates adjusted for the same covariables and including the AUC of CRP (Model 2), calculated by averaging over 1,000 bootstrap samples and non-parametrically obtaining $95 \%$ confidence intervals.

"inflamed" delirium (i.e. acute systemic inflammation at the onset of delirium) and long-term self-reported cognitive problems. Nevertheless, the sample size of this study was small $(\mathrm{n}=52)$ and inflammation was less well defined using systemic inflammatory response syndrome criteria at delirium onset. ${ }^{29,30}$ Using systemic inflammatory response syndrome criteria to define acute systemic inflammation is not advisable, as it has low sensitivity, and insufficient face and construct validity. ${ }^{30,31}$

We used a cumulative measure for inflammation during ICU stay (i.e., the AUC of the daily CRP levels) since we were interested in the effect of the total burden of inflammation on the association between delirium and long-term self-reported cognitive problems. We did not take the temporal association between inflammation and delirium into account, and therefore did not investigate the causal influence of inflammation related to delirium, and subsequently, self-reported cognitive outcome. It is plausible that the effect of acute systemic inflammation on longterm self-reported cognitive problems is more pronounced in patients with multiple days of delirium than in those with only a single delirium day, as the absolute difference is larger. Nevertheless, this difference was not statistically significant and therefore this observation is merely speculative.

Our large prospective cohort study has several strengths. The study population consisted of mixed medical-surgical ICU patients, which contributes to the generalizability of our results. The validated delirium assessment conducted by research nurses reduced the risk of misclassifying subjects. ${ }^{13}$ The response rate was relatively high thanks to careful follow-up of subjects using 
a postal reminder and follow-up phone calls. Furthermore, similar results were found in secondary analyses with multiple imputation, indicating that the results were robust. Also, the multivariable analyses were adjusted for important potential confounders, such as age, which is associated with a higher risk of delirium and may be associated with increased inflammation. Some potential limitations of our study should also be addressed. Differentiation between sedation-related and non-sedation related delirium could not be made in our cohort. Nevertheless, it is plausible that a single day of delirium during ICU stay reflects, at least in part, the rapidlyreversible sedation-related delirium. It could be that the sample of subjects with a single day of ICU delirium was too small to demonstrate any association. Furthermore, our results cannot directly be compared with studies that used a neuropsychological test battery, as the CFQ estimates subjective self-reported cognitive problems. Also, we did not have a baseline assessment of cognitive functioning and therefore cannot be sure that 1 year self-reported cognitive problems are solely due to prolonged ICU delirium or that subjects experienced prolonged ICU delirium because they already had underlying cognitive problems. In future studies, measuring baseline cognitive functioning is recommended. We also did not have information about events after ICU discharge, which may have influenced cognitive outcome, such as delirium after ICU discharge. As with all observational studies, residual confounding might have occurred. Hence, we could not causally relate ICU delirium to long-term self-reported cognitive problems. The CRP is a well-acknowledged marker of inflammation. However, it is nonspecific and not validated as a marker for neuroinflammation. For future research it may be interesting to focus on neuroinflammation-specific markers. It is also important to realize that, as the domain of our study was subjects alive 1 year after ICU discharge, no inference can be made to subjects who died earlier.

\section{CONCLUSION}

In conclusion, multiple days of ICU delirium was associated with long-term self-reported cognitive problems. The cumulative exposure to systemic inflammation did not alter this association, which suggests that delirium in the context of little inflammation is also detrimental.

\section{ACKNOWLEDGEMENTS}

Funding Source: none.

Conflict of Interest: The editor in chief has reviewed the conflict of interest checklist provided by the authors and has determined that the authors have no financial or any other kind of personal conflicts with this paper.

Author Contributions: AEW contributed to the design of the study, carried out the main analyses, interpreted the data and wrote the manuscript. LMP, DSV, and AJCS assisted substantially in the design of the study, the analysis, and critically revised the manuscript for important intellectual content. IJZ, DWL, DD, and OLC also participated substantially in the conception and design, participated considerably in its design and coordination, and reviewed the manuscript critically. WP made a substantial contribution to the acquisition of the data by constructing the dataset, performed part of the analyses, and reviewed the manuscript critically as well. All authors read and approved the final manuscript.

Sponsor's Role: No sponsor had any role in the design, methods, subject recruitment, data collections, analysis or preparation of the paper.

\section{REFERENCES}

1. Ehlenbach WJ, Hough CL, Crane PK et al. Association between acute care and critical illness hospitalization and cognitive function in older adults. JAMA 2010;303:763-770.

2. Iwashyna TJ, Ely EW, Smith DM et al. Long-term cognitive impairment and functional disability among survivors of severe sepsis. JAMA 2010;304:1787-1794

3. Pandharipande PP, Girard TD, Jackson JC et al. Long-term cognitive impairment after critical illness. N Engl J Med 2013;369:1306-1316.

4. Van den Boogaard M, Schoonhoven L, Evers AW et al. Delirium in critically ill patients: Impact on long-term health-related quality of life and cognitive functioning. Crit Care Med 2012;40:112-118.

5. Girard TD, Jackson JC, Pandharipande PP et al. Delirium as a predictor of long-term cognitive impairment in survivors of critical illness. Crit Care Med 2010;38:1513-1520.

6. Wolters AE, van Dijk D, Pasma W et al. Long-term outcome of delirium during intensive care unit stay in survivors of critical illness: A prospective cohort study. Crit Care 2014;18:R125.

7. Cerejeira J, Firmino H, Vaz-Serra A et al. The neuroinflammatory hypothesis of delirium. Acta Neuropathol 2010;119:737-754.

8. Cunningham C. Systemic inflammation and delirium: Important co-factors in the progression of dementia. Biochem Soc Trans 2011;39:945-953.

9. Van Gool WA, van de Beek D, Eikelenboom P. Systemic infection and delirium: When cytokines and acetylcholine collide. Lancet 2010;375:773775 .

10. MacLullich AMJ, Beaglehole A, Hall RJ et al. Delirium and long-term cognitive impairment. Int Rev Psychiatry 2009;21:30-42.

11. Qin L, Wu X, Block ML et al. Systemic LPS causes chronic neuroinflammation and progressive neurodegeneration. Glia 2007;55:453-462.

12. Patel SB, Poston JT, Pohlman A et al. Rapidly reversible, sedation-related delirium versus persistent delirium in the intensive care unit. Am J Respir Crit Care Med 2014;189:658-665.

13. Zaal IJ, Tekatli H, van der Kooi AW et al. Classification of daily mental status in critically ill patients for research purposes. J Crit Care 2015;30:375-380.

14. Sessler CN, Gosnell MS, Grap MJ et al. The Richmond agitation sedation scale: Validity and reliability in adult intensive care unit patients. Am J Respir Crit Care Med 2002;166:1338-1344.

15. Ely EW, Margolin R, Francis J et al. Evaluation of delirium in critically ill patients: Validation of the confusion assessment method for the intensive care unit (CAM-ICU). Crit Care Med 2001;29:1370-1379.

16. Merckelbach H, Muris P, Nijman $\mathrm{H}$ et al. Self-reported cognitive failures and neurotic symptomatology. Pers Individ Diff 1996;20:715-724.

17. Broadbent DE, Cooper PF, Fibgerald P et al. The Cognitive Failures Questionnaire (CFQ) and its correlates. Br J Clin Psychol 1982;21:1-16.

18. Kastaun S, Gerriets T, Schwarz NP et al. The relevance of postoperative cognitive decline in daily living: Results of a 1-year follow-up. J Cardiothorac Vasc Anesth 2016;30:297-303.

19. van Vliet $M$, van den Boogaard M, Donnelly $\mathrm{P}$ et al. Long-term health related quality of life following intensive care during treatment for haematological malignancies. PLoS ONE 2014;9:e87779.

20. Standage SW, Wong HR. Biomarkers for pediatric sepsis and septic shock. Expert Rev Anti Infect Ther 2011;9:71-79.

21. Matthews JNS, Altman DG, Campbell MJ et al. Analysis of serial measurements in medical research. BMJ 1990;300:230-235.

22. Quan H, Li B, Couris CM et al. Updating and validating the Charlson comorbidity index and score for risk adjustment in hospital discharge abstracts using data from 6 countries. Am J Epidemiol 2011;173:676682.

23. Zimmerman JE, Kramer A, McNair DS et al. Acute physiology and chronic health evaluation (APACHE) IV: Hospital mortality assessment for today's critically ill patients. Crit Care Med 2006;34:1297-1310.

24. Vincent J, de Mendonca A, Cantraine F et al. Use of the SOFA score to assess the incidence of organ dysfunction/failure in intensive care units: 
Results of a multicenter, prospective study. Working group on "sepsisrelated problems" of the European Society of Intensive Care Medicine. Crit Care Med 1998;26:1793-1800.

25. Zaal IJ, Devlin JW, Peelen LM et al. A systematic review of risk factors for delirium in the ICU. Crit Care Med 2015;43:40-47.

26. Engels JM, Diehr P. Imputation of missing longitudinal data: A comparison of methods. J Clin Epidemiol 2003;56:968-976.

27. Mackinnon DP, Fairchild AJ, Fritz MS. Mediation analysis. Annu Rev Psychol 2007;58:593.

28. Rubin DB, Schenker N. Multiple imputation in health-care databases: An overview and some applications. Stat Med 1991;10:585-598.

29. Van den Boogaard M, Kox M, Quinn KL et al. Biomarkers associated with delirium in critically ill patients and their relation with long-term subjective cognitive dysfunction; Indications for different pathways governing delirium in inflamed and noninflamed patients. Crit Care 2011;15:R297.

30. Kaukonen KM, Bailey M, Pilcher D et al. Systemic inflammatory response syndrome criteria in defining severe sepsis. N Engl J Med 2015;372:16291638.

31. Klein Klouwenberg PMC, Ong DSY, Bonten MJM et al. Classification of sepsis, severe sepsis and septic shock: The impact of minor variations in data capture and definition of SIRS criteria. Intensive Care Med 2012;38:811-819.

\section{SUPPORTING INFORMATION}

Additional Supporting Information may be found in the online version of this article:

Appendix S1. Characteristics of the 1-year ICU survivors; Groups divided in complete cases, partial responders, nonresponders and nonreceivers.

Appendix S2. Association between delirium and Cognitive Failures Questionnaire (CFQ) and the effect of systemic inflammation: Result after multiple imputation.

Please note: Wiley-Blackwell is not responsible for the content, accuracy, errors, or functionality of any supporting materials supplied by the authors. Any queries (other than missing material) should be directed to the corresponding author for the article. 